Automatic construction of restriction site maps

William R.Pearson

Department of Molecular Biology and Genetics, Johns Hopkins University School of Medicine, Baltimore, MD 21205, USA

Received 14 September 1981

ABSTRACT

A computer program is described which constructs maps of restriction endonuclease cleavage sites in DNA molecules, given only the fragment lengths. The program utilizes fragment length data from single and double restriction enzyme digests to generate maps for linear or circular molecules. The search for a map can be limited to the unknown (insert) region of a recombinant phage or plasmid. Typical restriction maps with four or five enzymes which cut at three to five unknown sites can be calculated in a few minutes.

\title{
INTRODUCTION
}

Restriction enzymes are among the most powerful tools of molecular genetics. Together with recombinant DNA techniques, they have given biologists the ability to explore genes with an ease undreamed of ten years ago. Usually soon after a recombinant clone containing a DNA fragment of interest is identified, experiments are done to map its restriction sites. A number of techniques have been developed for ordering restriction fragments $(1,2,3,4)$. Preliminary maps can of ten be constructed from single and double restriction enzyme digestions by ordering and aligning the single enzyme digestion fragments to agree with the double enzyme digestions. However, in many cases mapping requires more complex and time-consuming experiments.

In this paper we present computer programs that rapidly find restriction maps of circular and linear molecules. The programs search for a map in much the same way we do by hand - permuting the single enzyme fragments and aligning them until their overlap agrees with the double enzyme digestion data. Unlike most of us, however, the program finds all of the possible arrangements of fragments which agree with the data, and a molecule can often be mapped in a matter of minutes. 


\section{MAPPING STRATEGY}

As Figure 1 illustrates, the program first considers the pair of fragment sets from the first two restriction digests. For each possible ordering of the first set of fragments, every possible ordering of the second set of fragments is evaluated. If the restriction map is partially known, only fragments in the unknown region are permuted. Possible solutions are evaluated by calculating a hypothetical double enzyme digest and comparing the calculated double digest to the real double digest data. The hypothetical and experimental data are compared by sorting the lengths in decreasing order (analogous to running both sets of fragments on a "gel"). Then the squares of the differences of the largest fragment, the next largest and so on to the smallest double digest fragments are summed. This evaluation function has the advantage of giving more weight to the longest fragments. It is not affected by imprecise data on very short fragments. For example, the data in Figures 2 and 3 are from a plasmid which contains a short Pstl fragment (60 base pairs). The program maps the larger fragments correctly even though the 60 base pair fragment is omitted. Including the data for the short fragment does not affect the map for the larger fragments.

An earlier program, GAl, has been described which uses a different algorithm for calculating restriction maps (5). The algorithm described here can solve more difficult problems much more rapidly. GAl considered many of the permutations of the double-digest fragments. This algorithm only permutes the single-digest fragments. Since $A$ factorial times $B$ factorial is much smaller than $(A+B)$ factorial, this algorithm is much faster, and still considers all the possible maps which fit the data equally well.

The process of overlapping restriction digests of circular molecules is more difficult. While there is always a left most ( 0 offset) fragment in a digest of a linear molecule, circular molecules are more difficult to align. MAPCIR uses the NEW FRAGment double-digest fragment length data to offset each pair of restriction digests. The NEW FRAGment is the length of a double-digest restriction fragment which is not found in either of the single-enzyme digestions. It has the restriction site from the first enzyme on one end and the site from the second enzyme on the other end. By overlapping the restriction fragments from the first enzyme with the fragments of the second of fset by the NEW FRAGment length, the proper alignment can be found. 
A. Permutation generation
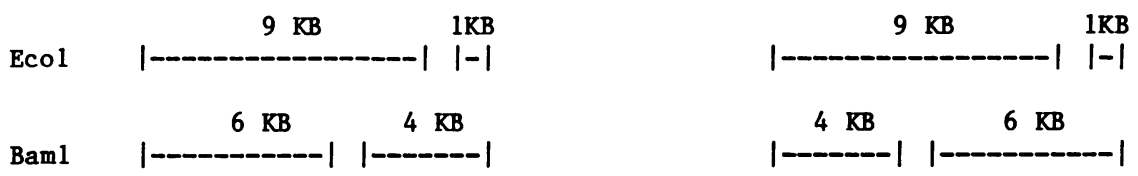

B. Calculation of hypothetical double digest fragments

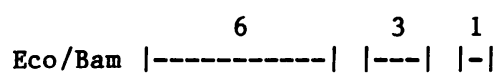

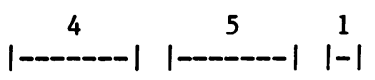

C. Comparison of hypothetical and actual double digest fragments hypothetical actual hypothetical

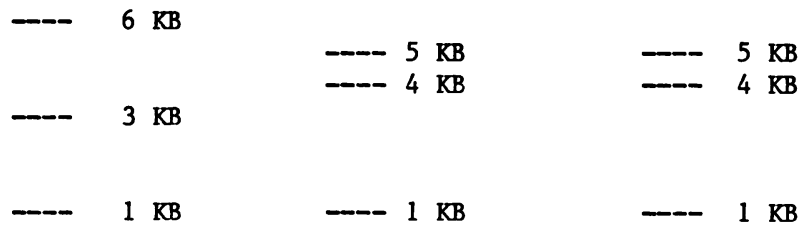

D. Error calculation

$$
\begin{array}{cc}
{ }^{2}(6-5)^{2}+(3-4)^{2}+(1-1)^{2} & (5-5)^{2}+(4-4)^{2}+(1-1)^{2} \\
=2 & =0
\end{array}
$$

FIGURE 1: An algorithm for mapping a linear molecule.

A simple example of a linear molecule is shown. The unknown DNA has been digested with two enzymes, EcoRl and BamHl and with EcoRl and BamHl together. A. The two possible permutations of the BamHl digest are compared with the EcoRl digest. Only one permutation of the EcoRl digest is considered since the second is just the reverse of the first. B. Hypothetical EcoRl/BamHl double digests are calculated for each of the possible maps. C. The double digest fragments are sorted from largest to smallest ("run on a gel") and compared with the double digest data. D. The squared errors are calculated. The right-hand solution has a lower error and is judged correct. 
If the differences between the calculated and actual double-digest fragment lengths are less than the ERROR criterion, the program moves on to the next restriction enzyme. Each permutation of the next single-enzyme restriction fragments is again evaluated against the ordering of the first enzyme. If a possible solution is found, it is checked against all of the earlier digest orderings using the ERROR*EFACT criterion. When all of the enzyme digests have been considered, the set of all the orderings is saved for later display.

The ERROR criterion weeds out many incorrect solutions, so that only a small fraction of the possible fragment orderings are evaluated further. For example, a three enzyme problem with 3, 4 and 5 fragments/enzyme would require at least 3 ! $\times 4$ ! X 5 ! or 17,280 evaluations if all possible solutions were considered. In the ideal case the ERROR criterion would consider only one solution of the 3 ! $\times 4$ ! problem against the 5 ! possible orderings of the third enzyme. In this case, only 3 ! X 4 ! + 5! or 264 solutions would need to be evaluated. In actual practice, an intermediate number of solutions is evaluated completely.

The order in which the different enzyme digestions are considered is very important. In the example above, if the enzymes were considered in order $5,4,3$, the minimum number of evaluations would increase to 5 ! $X 4 !+3$ ! or 2880 . The importance of the ERROR criteria and order of enzymes increases dramatically with the number of fragments. A fourth enzyme which made six cuts would increase the number of solutions evaluated four fold if it were entered last, but by more than 16 fold if it were entered first.

Limiting the scope of the search to the unknown insert region of a recombinant vector also speeds the solution dramatically. Many large molecules such as lambda phage are already partially mapped. Limiting consideration of the RI fragments of Charon 4A to the internal region decreases the number of permutations by a factor of 20 , from 120 (5!) to $6(3 !)$, in an example below.

In tests on a large timesharing computer the overlapping and sorting algorithm evaluted a solution in about $2 \mathrm{msec}$. The longest test we have tried was an unmapped circular molecule cut by five enzymes generating 1, 2, 5, 5 and 6 fragments/enzyme. This problem required about 2 minutes of computer time when a low ERROR criterion was used. 


\section{USING THE PROGRAMS}

Three programs have been written for calculating restriction maps. One of the programs, DIGFIL, is used to build a file of restriction digest data. The other two programs, MAPCIR and MAPLIN, actually permute the digest data and calculate the best restriction maps. Solving a restriction map problem involves running DIGFIL to enter the data, and then using MAPCIR or MAPLIN to analyze the data and display a map.

Figure 2 shows an example using DIGFIL to enter restriction data. The responses which were typed in are underlined. First, the program QDIGFIL ; a program for entering restriction data

DATA FILES FOR RESTRICTION MAPPING.

O-CIRCLE, 1-LINEAR: 0

TYPE UP TO 10 ENZYME NAMES, 4 CHARS/NAME 1/LINE

END WITH BLANK LINE

1: Baml

2: $\overline{\mathrm{Bg} 12}$

3: $\overline{\text { Pst1 }}$

4: $\quad$; no more enzymes

SINGLE DIGEST FRAGMENT LENGTHS FOR EACH ENZYME

Baml: $3.7601 .380 \quad 0.420$

Bg12: 5.550

Pst $1: \underline{4.320} \quad 0.430 \quad 0.390 \quad 0.340$

XOFF ( $-1=$ UNKNOWN), IBEG, IEND OF UNKNOWN REGION

Baml: 0.375 ; XOFF is known, IBEG and IEND are not used

Bg12: -1 ; XOFF is not known, so -1 is entered

Pst $1: \overline{3.612}$

ENTER DOUBLE DIGEST FRAGMENT LENGTHS

THEN DOUBLE DIGEST CREATED FRAGMENT ON NEW LINE

Baml Bg12: $3.760 \quad 1.380 \quad 0.300 \quad 0.140$

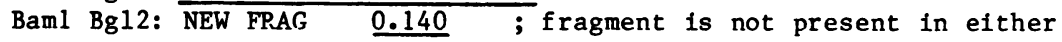

Baml Pst1: $\begin{array}{llll}3.240 & 1.080 & 0.430 & 0.390 \quad 0.280\end{array}$

Baml Pst 1: NEW FRAG $\underline{3.240}$

Bg12 Pst1: $4.320 \quad 0.430 \quad 0.340 \quad 0.250 \quad 0.140$

Bg12 Pst 1: NEW FRAG $\underline{0.140}$

ENTER DATA FILE NAME(A10): PGT55E.DAT

TITLE LINE: DGT55 glutathione S-transferase 16-Jan-81

STOP

FIGURE 2: Entering restriction fragment data

The DIGFIL program was used to enter restriction data for a circular molecule. The data is from a cDNA clone inserted in the PBR 322 at the Pstl site. Typed in responses are underlined. Additional comments preceded by a semicolon ';' are included in the figure. 
requests the topology of the molecule to be mapped ( 0 for circular, 1 for linear). It then asks for the names of the restriction enzymes used. The names may be up to four characters long (e.g. HAE3 or hpa2). A blank line indicates the end of the list. Up to 10 restriction enzymes may be considered at the same time, but since the program does not provide partial solutions, it is safer to start with four or five enzymes and gradually build a restriction map. Each enzyme may generate up to 20 restriction fragments.

The order of the restriction enzyme digests is important. Enzymes which make a small number of cuts should be entered before enzymes that make a large number of cuts. In addition, the single and double enzyme digestions must be related to one another. If double digests between enzymes 1-2, 2-3 and 4-5 were used without a 1-4 or 2-4, there would be no way for the program to use the 4-5 data to confirm the maps of enzymes 1,2 and 3.

After the restriction enzyme names are entered, the program requests the single enzyme digestion data for each enzyme. Fragment lengths can be entered in any units - nucleotides, kilobases (KB), percent - and, with one exception, in any order. The order of the fragments is only important if part of the restriction map is known. For example, an EcoRI digest of Charon $4 \mathrm{Amight}$ be entered as

$\begin{array}{lllll}19.8 & 14.2 & 5.6 & 3.4 & 10.8\end{array}$

to specify the left (19.8) and right (10.8) end fragments (6).

After all of the single enzyme data are entered, some additional data are requested. FIrst IBEG and IEND, the indices of the fragments to be mapped, are entered. IBEG is the index of the first unmapped restriction fragment ( 14.2 in the example above) and IEND is the index of the last unmapped fragment (3.4 in the example). By entering 2, 4 one specifies that the fragments at position $2(14.2), 3(5.6)$ and $4(3.4)$ should be permuted to find the restriction map while the first and last fragments remain in place. If nothing is known about the location of any of the restriction fragments, the line is left blank.

The circular molecule in Figure 2 is more complex. In addition to IBEG and IEND, another parameter, XOFF, is requested. XOFF is a coordinate of a known restriction site for the enzyme in question. For example, if one were mapping a recombinant plasmid using pBR322 as the vector, the XOFF's for EcoRI, BamHI and PstI would be $0.0,0.375$ and $3.612 \mathrm{~KB}(7)$. In Figure 2 the XOFF values for Baml (0.375) and Pst 1 
(3.612) were entered. If there are several known sites for a restriction enzyme, then any one of those sites may be entered (e.g. ECR2 at 0.129 or 1.057$)$. If the location of a restriction site is not known (e.g. Bg12 which does not cut in pBR322) -1 for UNKNOWN is used. IBEG and IEND were not entered in Figure 2. However, if Hae3 restriction fragments were mapped, it would be important to enter the pBR322 Hae 3 fragments in order followed by the fragments from the insert. IBEG and IEND could then be used to limit the permutations to the unknown region.

NOTE: while IBEG and IEND may be left blank (ignored) for most circular molecules, XOFF cannot be. A blank $(0.0)$ XOFF aligns each digest at 0.0 , which is not usually the case. To prevent misalignment, an XOFF of -1 (UNKNOWN) must be used.

The double enzyme digestion data are now entered. The program prompts for each pair of enzymes. If data are not avallable for a particular enzyme, the line may be left blank and that pair will be ignored in the search for the map. (But the pairs of enzymes must be related, as mentioned earlier.) If the molecule is circular, an additional value (NEW FRAG) is requested. The NEW FRAGment length is the length of any fragment appearing in the double digest data but not in either set of single digest data. In the Baml Bg12 double digest in Figure 2, the 3.760 and $1.380 \mathrm{~kb}$ fragments were present in the Baml single digest, but the 0.300 and $0.140 \mathrm{~kb}$ fragments were generated in the double digest. Either 0.300 or 0.140 could be used as a NEW FRAG. A NEW FRAGment value must be entered.

After all the data are entered the program requests a filename. This name is used to refer to the data file in MAPCIR and MAPLIN. The program also saves a title, which may be any line of characters.

After the data file has been built, the mapping program is used. Figure 3 shows a sample run of MAPCIR on the circular data of Figure 2. If a solution is found, the program first summarizes the fragment length data. The summary can be used to calculate the exact locations of restriction sites from the maps. The fragments are sorted in decreasing order, with the (A) fragment first, then the (B) fragment, and so on. As many as eight restriction map solutions are then printed. The program finds all of the solutions which agree with the data within the ERROR limits. If more than elght solutions are within the ERROR limits, the best eight are displayed. In Figure 3, four of the eight solutions are shown. One map is shown for each restriction enzyme. The 


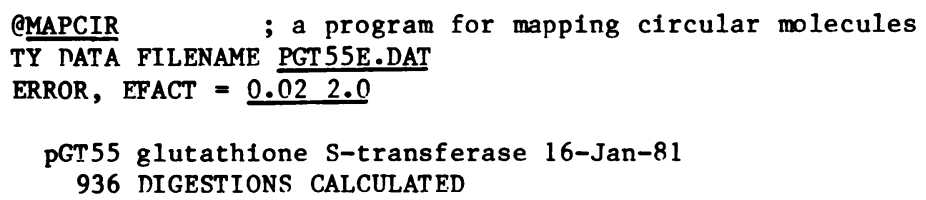

FIGURE 3: A restriction map calculated by the MAPCIR program MAPCIR was used to calculate a restriction map based on the data in Figure 2. Only the hest four of the eight solutions found are included. The first solution with the lowest $T$ FRPOR is the correct map of the plasmid. The map required 6 seconds of computer time. 
restriction sites are denoted by numbers, 1 for the first enzyme, 2 for the second, etc. The identity of each single digest fragment is marked by the letter $(A, B, C)$ corresponding to the fragment in the summary table. Circular maps include a $20 \%$ overlap so that the left end of the map $(0.0-0.2)$ is repeated at the right end $(1.0-1.2)$. The location of 1.0 is marked with a vertical bar ' $\mid$ '. Because of the limited resolution of the printed map (2\%), restriction fragment letters will sometimes be printed at the location of the restriction site and very small fragments may not appear. A list of the restriction fragments in map order is displayed after the map, so that short fragments which may be invisible on the map can be identified. This list is not aligned, but does give the map order of the fragments.

Two error values, $T$ ERROR and $D$ ERROR are calculated for each map. $T$ ERROR is the average total error for all of the single digest pairs (Baml - Bg12, Baml - Pst1 and Bg12 - Pst1 in Figure 3). D ERROR is the average error for the aligned pairs (Baml - Bg12 and Baml - Pst1). The D ERROR is the value limited by the ERROR value specified before the mapping begins. The restriction maps are presented in rank from best to worst by T ERROR. Each restriction map has several parts. The map of a circular molecule starts with a set of offset coordinates. Linear maps do not use offsets, because the maps are aligned at the ends of the molecule. In Figure 3, the coordinates are 0.375 for Baml, 4.27 for Bg12 and 3.61 for Pstl. The offset coordinates for Baml and Pstl are the same as the XOFF values given when the data was entered with DIGFIL. The offset for the Bg12 site was unknown, however, and has been calculated. The offset value is the distance between the 0.0 coordinate of the molecule and the left most restriction site for the enzyme. The location of any restriction site can be calculated using the offset and the lengths of the restriction fragments. For example, the left end of the Baml $C$ fragment is at an offset of 0.375 plus the length of fragment A (from the summary table, 3.760 ) or $4.135 \mathrm{~KB}$.

In Figure 3, four of the eight sets of maps calculated are shown. Each of the sets of maps has a different $T$ ERROR, and the map with the best $T$ ERROR is known to be correct from additional evidence. The difference between the best and second best solution is the location of the Bg12 site. The offset coordinate of the Bg12 site is $4.27 \mathrm{~KB}$ in the first solution and $4.41 \mathrm{~KB}$ in the second. This shift in the Bg12 site almost doubles the $T$ ERROR but does not affect the $D$ ERROR. The D ERROR 
is unaffected because it does not include the Bg12 - Pstl comparison which fixes the position of the $\mathrm{Bg} 12$ site. The second pair of solutions differs from the first pair in the order of the Pstl fragments ( B C D A vs. C B D A). Sometimes, two or more maps will have an identical T ERROR. In these cases, the available data do not distinguish between the possibilities. Often there will be several maps with the same order of fragments but slightly different offsets and errors. Usually the differences are small and the map has been found.

MAPLIN is used to map linear molecules. The programs ask for the name of the data file made by DIGFIL (PGT55E in the example shown) and then request two error parameters ERROR and EFACT. If ERROR and EFACT are left blank, the default values ERROR $=0.025$ and EFACT $=2$ w111 be used. The program then permutes the data and finds the best alignment of the single enzyme digestions that agrees with the double enzyme digestion data.

ERROR and EFACT are two parameters that limit the different possible restriction maps to be searched. The mapping programs find solutions by comparing the double digest fragments of hypothetical maps to the experimental data. If the difference between the hypothesis and data is too great, that permutation of the fragments is no longer considered. The fewer incorrect permutations considered, the faster the solution is found. For maps with enzymes which make more than 3 or 4 fragments, the ERROR limit should be reduced to 0.01 or 0.005 and a solution attempted. It is much faster to look for a solution with a low ERROR limit and fail and try again than to examine many thousands of incorrect permutations. For example, a test data file of four enzymes each of which makes three fragments takes 23 seconds to solve with the default 0.025 ERROR but only 2 seconds with an ERROR limit of 0.005 .

EFACT is a factor which modifies the ERROR limit for certain double digest comparisons. In analyzing the single and double enzyme data, each digest is compared with the first restriction digest $(1-2,1-3)$ and the ERROR criterion used. However, since the enzyme 2 and 3 restriction digests have been aligned with the data from enzyme 1, the 2-3 map may be correct but poorly aligned. Thus a less strict criterion, ERROR*EFACT (or $0.025 * 2=0.05$ ) is used for these out of alignment comparisons. 


\section{DISCUSSION}

The programs DIGFIL, MAPCIR and MAPLIN provide a quick method for generating simple restriction maps. The algorithm evaluates only the ordering necessary to find a solution, but will find all alternate solutions of similar accuracy. Exhaustively searching for alternate solutions manually is almost impossible.

The programs are good at examining the small number of restriction fragments generated in initial maps of recombinant molecules. Because of the factorial explosions encountered in the search, initial use of a digest with more than 7 or 8 unmapped fragments (5040 or 40,320 permutations) is not very practical. However, if a map is well known after digestion with enzymes that cut 3,4 or 5 times, adding 8 fragment data will not be too time-consuming (another 40,000 solutions must be evaluated).

The programs described are written in FORTRAN for the DECsystem-10 computer. The programs may be used through the MOLGEN project on the Stanford SUMEX computer system. Coples of the program are avallable from the author.

This research was supported by a grant from the National Institute of General Medical Sciences to John F. Morrow. W.R.P. was supported by N.I.H. Grant 2 T32 CA 09139 and thanks the MOLGEN project at Stanford for the opportunity to make the program generally avallable.

\section{REFERENCES}

1 Nathans, D. and Smith, H. O. (1975) Ann. Rev. Biochem. 44,273-293

2 Smith, H. 0. and Birnstiel, M. L. (1976) Nuc. Acids Res . 3,2387-2398

3 Parker, R. C., Watson, R. M. and Vinograd, J. (1977) Proc. Natl. Acad. Sc1. US 74,851-855

4 Sato, S., Hutchison, C. A., and Harris, J. I. (1977) Proc. Nat1. Acad. Sc1. US 74,542-546

5 Stefik, M. (1978) Artificial Intelligence 11,85-114

6 Daniels, D. L., de Wet, J. R. and Blattner, F. R. (1980) J. Virol. 33, 390-400

7 Sutcliffe, J. G. (1979) Cold Spring Harbor Symp. Quant. Biol. 43,77-90 